Computer Vision, Graphics, and Pattern Recognition Group

Department of Mathematics and Computer Science

University of Mannheim

D-68131 Mannheim, Germany

Reihe Informatik

$5 / 2004$

Binary Tomography by Iterating Linear Programs

S. Weber, C. Schnörr, T. Schüle, J. Hornegger

Technical Report 5/2004

Computer Science Series

June 2004

The publications of the CVGPR Group are listed under http://www.cvgpr. uni-mannheim.de 


\title{
BINARY TOMOGRAPHY BY ITERATING LINEAR PROGRAMS
}

\author{
Stefan Weber, ${ }^{1}$ Christoph Schnörr, ${ }^{1}$ Thomas Schüle, ${ }^{1,3}$ and \\ Joachim Hornegger ${ }^{2}$ \\ ${ }^{1}$ University of Mannheim \\ Dept. M\&CS, CVGPR-Group, D-68131 Mannheim, Germany \\ www.cvgpr.uni-mannheim.de \\ \{wstefan, schnoerr, schuele\}@uni-mannheim.de \\ ${ }^{2}$ Friedrich-Alexander University Erlangen-Nürnberg \\ Dept. CS, D-91058 Erlangen, Germany \\ www5.informatik. uni-erlangen.de \\ joachim@hornegger.de \\ ${ }^{3}$ Siemens Medical Solutions \\ D-91301 Forchheim, Germany \\ www. siemensmedical.com
}

\begin{abstract}
A novel approach to the reconstruction problem of binary tomography from a small number of $\mathrm{X}$-ray projections is presented. Based on our previous work, we adopt a linear programming relaxation of this combinatorial problem which includes an objective function for the reconstruction, the approximation of a smoothness prior enforcing spatially homogeneous solutions, and the projection constraints. We supplement this problem with an unbiased concave functional in order to gradually enforce binary minimizers. Application of a primal-dual subgradient iteration for optimizing this enlarged problem amounts to solve a sequence of linear programs, where the objective function changes in each step, yielding a sequence of solutions which provably converges.
\end{abstract}

Keywords: Discrete Tomography, Combinatorial Optimization, Linear Programming, D.C. Programming

\section{Introduction}

Discrete Tomography is concerned with the reconstruction of discrete-valued functions from projections. Historically, the field originated from several branches of mathematics like, for example, the combinatorial problem to determine binary matrices from its row and column sums (see the survey [10]). Meanwhile, 
however, progress is not only driven by challenging theoretical problems $[5,7]$ but also by real-world applications where discrete tomography might play an essential role (cf. [8, chapters 15-21]).

The work presented in this paper is motivated by the reconstruction of volumes from few projection directions within a limited range of angles. From the viewpoint of established mathematical models [11], this is a severely ill-posed problem. The motivation for considering this difficult problem relates to the observation that in some specific medical scenarios (see below), it is reasonable to assume that the function $f$ to be reconstructed is binary-valued. This poses one of the essential questions of discrete tomography: how can knowledge of the discrete range of $f$ be exploited in order to regularize and solve the reconstruction problem?

Medical Application. A potential application of discrete tomography in the field of medical imaging is the 3D reconstruction from Digital Subtraction Angiography (DSA) images. DSA is a common technique for separating contrast-filled vessels from the background. To this end, two images of the same scenery are taken, one with contrast-agent and another one without (see Figure 1). This results in low-noise projection images as input data for the reconstruction of a function which is assumed to be binary.
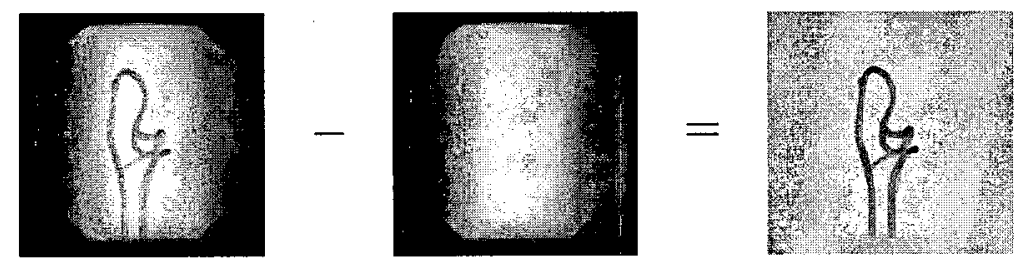

Figure 1. Illustration Digital Subtraction Angiography (DSA) imaging. A pair of images is taken from each projection direction, one (left) with and another one (center) without contrast agent. Subtraction of both images yields an image (right) that shows the distribution of the contrast agent only.

Problem Statement. The imaging geometry is represented by a linear system of equations $A x=b$. Each projection ray corresponds to a row of matrix $A$, and its projection value is the corresponding component of $b$. The row entries of $A$ represent the length of the intersection of pixels (voxels in the 3D case) of the (arbitrarily) discretized volume and the corresponding projection ray (see Fig. 2). This corresponds to the assumption that the function to be reconstructed is binary-valued, i.e. $x$ is a binary-valued vector. Each component $x_{i} \in\{0,1\}$ indicates whether the corresponding pixel (belongs to the reconstructed object, $x_{i}=1$, or not, $x_{i}=0$ (see Fig. 2). The reconstruction problem 
is to compute the binary indicator vector $x$ from the under-determined linear system of projection equations:

$$
A x=b, \quad x=\left(x_{1}, \ldots, x_{n}\right)^{\top} \in\{0,1\}^{n}
$$

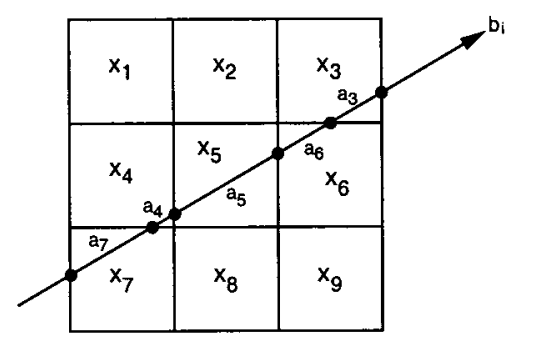

Figure 2. Discretization model leading to the algebraic representation of the reconstruction problem: $A x=b, x \in\{0,1\}^{n}$.

\section{Previous Work and Contribution}

Due to noise in the measurement vector $b$ when dealing with real data, (1) is likely to have no feasible solution. In order to take advantage of continuous problem formulations and numerical interior point methods, Fishburn et al. [4] considered the relaxation $x_{i} \in[0,1], i=1, \ldots, n$, and investigated the following linear programming approach for computing a feasible point:

$$
\min _{x \in[0,1]^{n}}\langle 0, x\rangle, \quad A x=b
$$

In particular, the information provided by feasible solutions in terms of additivity and uniqueness of subsets $\mathcal{S} \subset \mathbb{Z}^{n}$ is studied in [4].

Gritzmann et al. [6] introduced the following linear integer programming problem for binary tomography:

$$
\max _{x \in\{0,1\}^{n}}\langle e, x\rangle, \quad e:=(1, \ldots, 1)^{\top}, \quad A x \leq b,
$$

and suggested a range of greedy approaches within a general framework for local search. Compared to (2), the objective function (3), called best-innerfit $(B I F)$ in [6], looks for the maximal set compatible with the measurements. Furthermore, the formulation of the projection constraints is better suited to cope with measurement errors and noise.

In $[13,14]$, we studied the relaxation of (3) $x_{i} \in[0,1], \forall i$, supplemented with a standard smoothness prior enforcing spatial coherency of solutions

$$
\sum_{\langle i, j\rangle}\left(x_{i}-x_{j}\right)^{2}
$$


Here, the sum runs over all 4 nearest neighbors of the pixel grid (6 neighbors in the $3 \mathrm{D}$ case). In order to incorporate this prior into the linear programming approach (3), we used the following approximation by means of auxiliary variables $\left\{z_{\langle i, j\rangle}\right\}$ :

$$
\min _{x \in[0,1]^{n},\left\{z_{\langle i, j\rangle}\right\}}-\langle e, x\rangle+\frac{\alpha}{2} \sum_{\langle i, j\rangle} z_{\langle i, j\rangle}
$$

$$
\text { subject to } A x \leq b, \quad z_{\langle i, j\rangle} \geq x_{i}-x_{j}, z_{\langle i, j\rangle} \geq x_{j}-x_{i}
$$

Contribution. A global minimizer of the linear program (5) can straightforwardly be computed using an interior point method. In [14] we showed that for sparse volume structures, like blood vessels in the brain, in principle, rather accurate 3D-reconstructions may result from solving (5), provided an additional user parameter determining the rounding $[0,1] \ni x_{i} \rightarrow\{0,1\}, \forall i$, is set properly in a postprocessing step.

To get rid of this parameter, we supplement (5) with a concave functional enforcing binary solutions $x \in\{0,1\}^{n}$. Applying a two-step subgradient minimization technique leads to a sequence of programs of type (5), whose solutions converge to a local binary-valued minimizer.

Our approach may be regarded as an alternative to $[9,1]$ where different techniques have been suggested for rounding solutions of relaxed optimization problems. Rather than rounding in a postprocessing step, we integrate both objective functionals for reconstruction and binary-valued solutions into a single optimization problem, and solve it with a suitable mathematical programming approach.

\section{Optimization Approach}

Our approach reads:

$$
\begin{gathered}
\min _{x \in[0,1]^{n},\left\{z_{\langle i, j\rangle}\right\}}-\langle e, x\rangle+\frac{\alpha}{2} \sum_{\langle i, j\rangle} z_{\langle i, j\rangle}+\frac{\mu}{2}\langle x, e-x\rangle \\
\text { subject to } A x \leq b, \quad z_{\langle i, j\rangle} \geq x_{i}-x_{j}, z_{\langle i, j\rangle} \geq x_{j}-x_{i}
\end{gathered}
$$

Compared to (5), we supplemented in (6) the concave functional

$$
\frac{\mu}{2}\langle x, e-x\rangle=\frac{\mu}{2} \sum_{i} x_{i}-x_{i}^{2}
$$

which is minimal at the vertices of the domain $[0,1]^{n}$. Furthermore, since it vanishes at $\{0,1\}^{n}$, it does not alter binary minimizers of the original problem. Our strategy is to choose an increasing sequence of values for $\mu$ and to minimize for each of them (6). 
Problem (6) is no longer convex, of course. To explain our approach for computing a minimizer, we put

$$
z:=\left(x^{\top}, \ldots, z_{\langle i, j\rangle}, \ldots\right)^{\top}
$$

and rewrite with a slight abuse of notation all constraints of (6)

$$
0 \leq x_{i} \leq 1, \quad A x \leq b, \quad z_{\langle i, j\rangle} \geq x_{i}-x_{j}, z_{\langle i, j\rangle} \geq x_{j}-x_{i}
$$

in the form

$$
A z \leq b,
$$

with $A, b$ re-defined accordingly. Using the notation

$$
\delta_{C}(z)= \begin{cases}0 & , z \in C \\ +\infty & , z \notin C\end{cases}
$$

for the indicator functions of a convex set $C$, problem (6) then reads:

$$
\min _{z} f(z)
$$

where (cf. definition (8))

$$
\begin{aligned}
f(z) & =-\langle e, x\rangle+\frac{\alpha}{2} \sum_{\langle i, j\rangle} z_{\langle i, j\rangle}+\frac{\mu}{2}\langle x, e-x\rangle+\delta_{K}(b-A z), \\
& =g(z)-h(z)
\end{aligned}
$$

$K=\mathbb{R}_{+}^{n}$ is the standard cone of nonnegative vectors, and

$$
\begin{aligned}
& g(z)=-\langle e, x\rangle+\frac{\alpha}{2} \sum_{\langle i, j\rangle} z_{\langle i, j\rangle}+\delta_{K}(b-A z), \\
& h(z)=\frac{\mu}{2}\langle x, x-e\rangle .
\end{aligned}
$$

Note that both functions $g(z)$ and $h(z)$ are convex, and that $g(z)$ is non-smooth due to the linear constraints.

To proceed, we need the following basic concepts [12] defined for a function $f: \mathbb{R}^{n} \rightarrow \bar{R}$ and a set $C \subset \mathbb{R}^{n}$ :

$$
\begin{aligned}
\operatorname{dom} f=\left\{x \in \mathbb{R}^{n} \mid f(x)<+\infty\right\} & \text { effective domain of } f \\
f^{*}(y)=\sup _{x \in \mathbb{R}^{n}}\{\langle x, y\rangle-f(x)\} & \text { (conjugate function) } \\
\partial f(\bar{x})=\{v \mid f(x) \geq f(\bar{x})+\langle v, x-\bar{x}\rangle, \forall x\} & \text { subdifferential of } f \text { at } \bar{x}
\end{aligned}
$$

We adopt from $[3,2]$ the following two-step subgradient algorithm for minimizing (11): 


\section{Subgradient algorithm:}

Choose $z^{0} \in \operatorname{dom} g$ arbitrary.

For $k=0,1, \ldots$ compute:

$$
\begin{gathered}
y^{k} \in \partial h\left(z^{k}\right) \\
z^{k+1} \in \partial g^{*}\left(y^{k}\right)
\end{gathered}
$$

The investigation of this algorithm in [2] includes the following results:

Proposition 1 ([2]) Assume $g, h: \mathbb{R}^{n} \rightarrow \overline{\mathbb{R}}$ be proper, lower-semicontinuous and convex, and

$$
\operatorname{dom} g \subset \operatorname{dom} h, \quad \operatorname{dom} h^{*} \subset \operatorname{dom} g^{*} .
$$

Then

(i) the sequences $\left\{z^{k}\right\},\left\{y^{k}\right\}$ according to (14), (15) are well-defined,

(ii) $\left\{g\left(z^{k}\right)-h\left(z^{k}\right)\right\}$ is decreasing,

(iii) every limit point $z^{*}$ of $\left\{z^{k}\right\}$ is a critical point of $g-h$.

\section{Reconstruction Algorithm}

We apply (14), (15) to problem (6). Condition (16) holds, because obviously $\operatorname{dom} g \subset \operatorname{dom} h$, and $g^{*}(y)=\sup _{z}\{\langle z, y\rangle-g(z)\}<\infty$ for any finite vector $y$.

(14) reads

$$
\begin{aligned}
y^{k} & =\nabla h\left(z^{k}\right) \\
& =\mu\left(x^{k}-\frac{1}{2} e\right)
\end{aligned}
$$

since

$$
\partial h(\bar{z})=\{\nabla h(\bar{z})\}
$$

if $h$ is differentiable [12]. To compute (15), we note that $g$ is proper, lowersemicontinuous, and convex. It follows [12] that

$$
\begin{aligned}
\partial g^{*}(\bar{y}) & =\left\{z \mid g^{*}(y) \geq g^{*}(\bar{y})+\langle z, y-\bar{y}\rangle, \forall y\right\} \\
& =\operatorname{argmax}_{z}\{\langle\bar{y}, z\rangle-g(z)\}
\end{aligned}
$$

which is a convex optimization problem. Hence, (15) reads:

$$
z^{k+1} \in \operatorname{argmin}_{z}\left\{g(z)-\left\langle y^{k}, z\right\rangle\right\}
$$

Inserting $y^{k}$ from (17), we finally obtain by virtue of (12), (9), and (8): 


\section{Reconstruction algorithm ( $\mu$ fixed)}

Choose $z^{0} \in \operatorname{dom} g$ arbitrary.

For $k=0,1, \ldots$, compute $z^{k+1}$ as minimizer of the linear program:

$$
\min _{x \in[0,1]^{n},\left\{z_{\langle i, j\rangle}\right\}}-\left\langle e+\mu\left(x^{k}-\frac{1}{2} e\right), x\right\rangle+\frac{\alpha}{2} \sum_{\langle i, j\rangle} z_{\langle i, j\rangle}
$$

subject to $A x \leq b, \quad z_{\langle i, j\rangle} \geq x_{i}-x_{j}, z_{\langle i, j\rangle} \geq x_{j}-x_{i}$

Here, $A x \leq b$ are the original constraints from (6).

In practice, we start with $\mu=0$ and repeat the reconstruction algorithm for increasing values of $\mu$, starting each iteration with the previous reconstruction $z^{k}$. This outer iteration loop terminates when $\forall i, \min \left\{x_{i}, 1-x_{i}\right\}<\varepsilon$.

Note that for $\mu=0$, we minimize (5), whereas for $\mu>0$ it pays to shift in (20) the current iterate in the direction of the negative gradient of the "binarization" functional (7). While this is an intuitively clear modification of (5), convergence of the sequence of minimizers of (20) due to proposition 1 is not obvious.

\section{Experimental Results}

We compare iterative linear programming (20), with the regularized best inner fit approach (5).

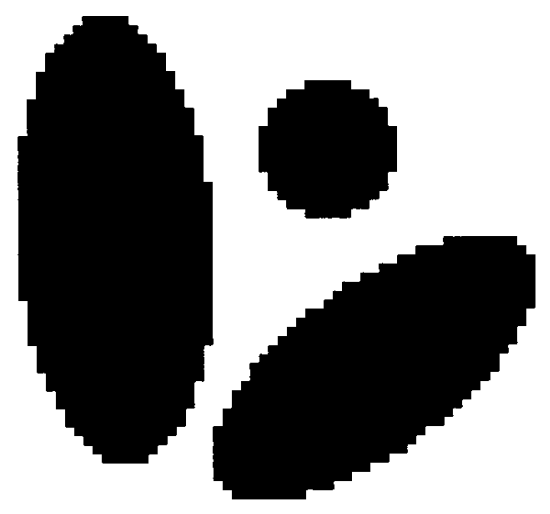

(a) Original, $64 \times 64$, experiment 1 .

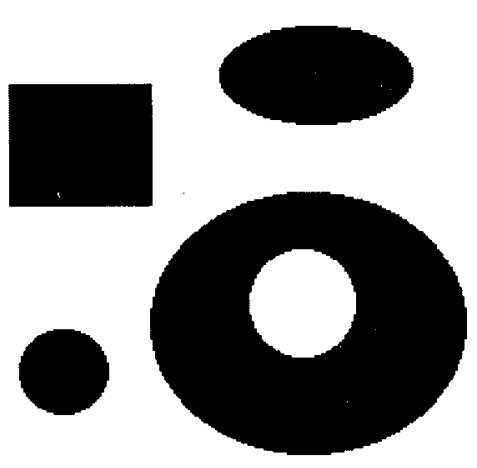

(b) Original, $256 \times 256$, experiment 2 .

Figure 3. From each image, 3 projections, $0^{\circ}, 45^{\circ}$, and $90^{\circ}$ were taken for setting up the two reconstruction problems used in our evaluation.

For evaluation purposes, we created two reconstruction problems from the images shown in figure 3. From each image, three projections were taken, 
$0^{\circ}, 45^{\circ}$, and $90^{\circ}$. Figure 4 shows the reconstruction results of the regularized best inner fit approach (5). This result illustrates that both reconstruction problems are not easy to solve due to the large area covered by the objects and the corresponding amount of self-occlusions.

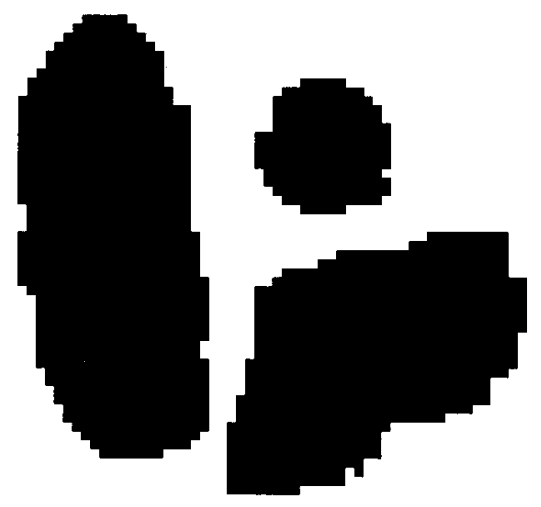

(a) Regularized BIF, experiment 1.

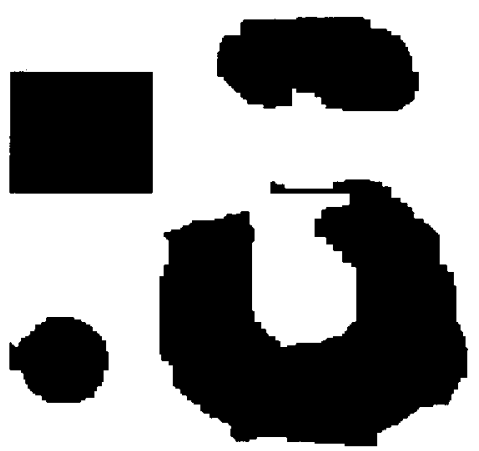

(b) Regularized BIF, experiment 2.

Figure 4. Results obtained by the regularized best inner fit approach (5).

Throughout all experiments, the parameter $\mu$ was initialized with 0 . After each iteration $\mu$ was increased by 0.1 in the first experiment and 0.05 in the second one. Further, the regularization parameter $\alpha$ was choosen as 0.5 in the first and 1.0 in the second experiment.

Comparison of the results for (20) and (5) in figures 5 and 7, respectively, shows the superior performance of the approach (20). The reason is that, through iterating the linear programs, rounding is not done as a separate postprocessing step, but during optimization, while taking into account the projection constraints. Figures 8 and 9 illustrate intermediate results for both reconstruction problems after different numbers of iterations. One can see how the solution converges towards a binary vector because of the increasing influence of the functional (7). Figure 6 further illustrates this process.

Concerning computation time, a single iteration (solving one LP) of the $64 \times 64$ image costs about 7 seconds, while it was about 4 minutes and 6 seconds for the $256 \times 256$ image.

\section{Conclusion and Further Work}

In this paper we have shown a new reconstruction approach based on linear programming for the problem of discrete tomography. Unlike other LP 


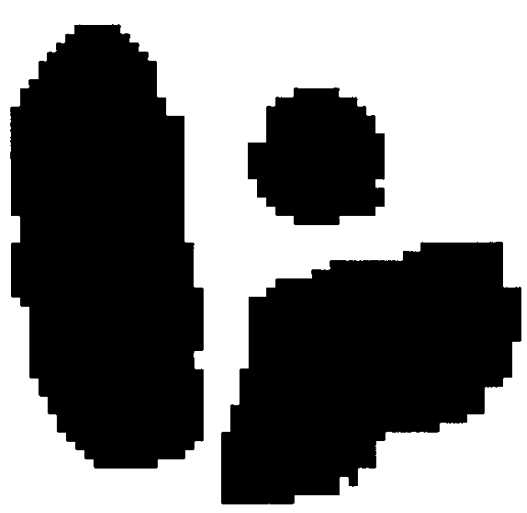

(a) Regularized BIF

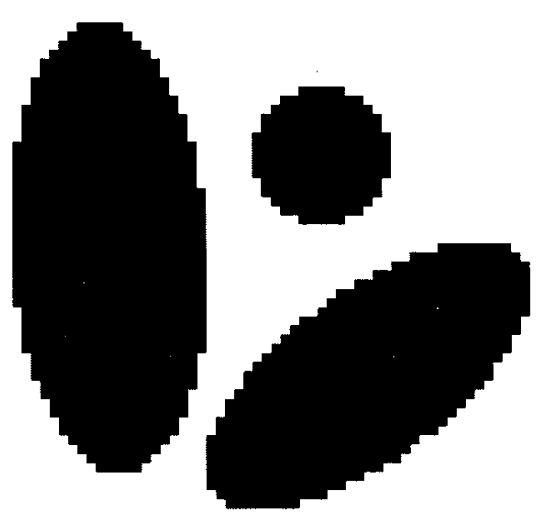

(b) Iterated LPs (10 Iterations).

Figure 5. Comparison between the regularized best inner fit approach and our approach proposed in this paper.

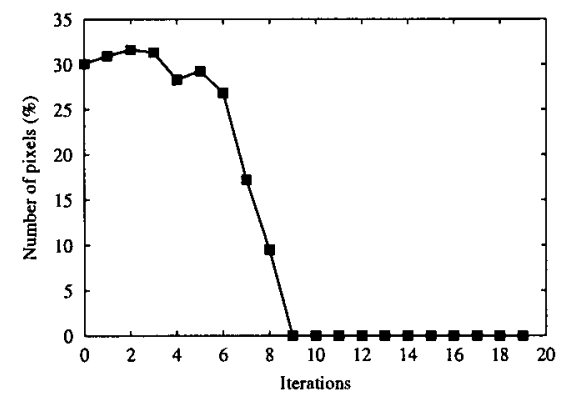

(a) First reconstruction problem.

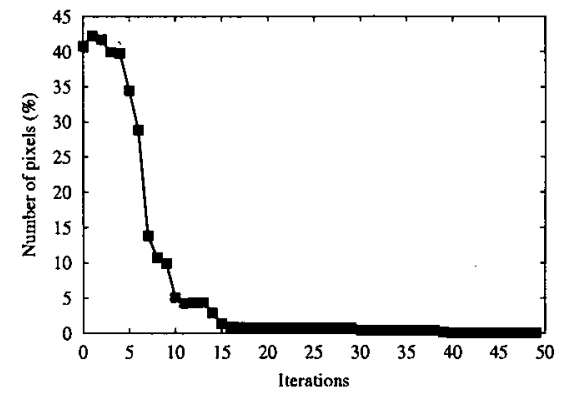

(b) Second reconstruction problem.

Figure 6. Both graphs show the percentage of non-binary pixels per iteration. The graph in (a) corresponds to the first reconstruction experiment and to the images shown in figure 8 . After 9 iterations the solution became binary which in this case was the original image. The graph in (b) shows the same data for the second experiment which is shown in figure 9. After 51 iterations the curve dropped down to $0.07 \%$. We simply set these tiny fraction of pixels to zero and terminate the algorithm.

methods, the rounding process is now explicitly done within the reconstruction process and not as a postprocessing step after the reconstruction. Hence, the problem constraints of the linear program do affect the rounding. On the other hand, one has to solve a sequence of LPs instead of a single one which of course leads to more computationally effort. However, the linear programs do 


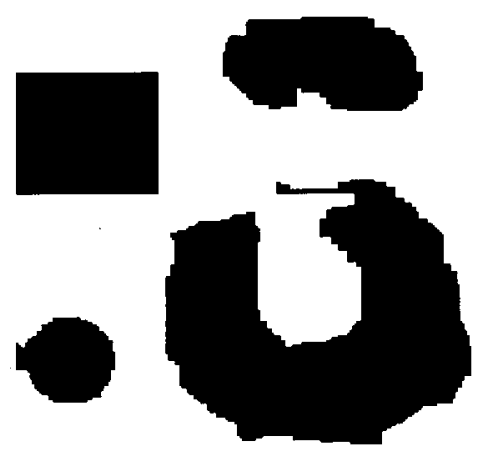

(a) Regularized BIF

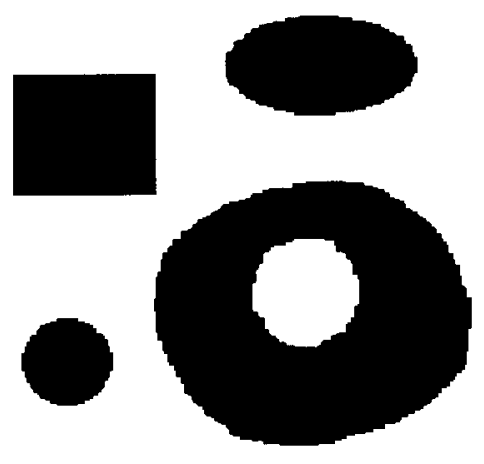

(b) Iterated LPs (51 Iterations).

Figure 7. Comparison between regularized BIF and iterating LPs for the second experiment. We terminated the iterated LPs after 51 iterations and set the remaining non-binary pixels $(0.07 \%)$ to zero in order to obtain a binary solution.

not differ too much from each other, as only the target vector $c$ has to be modified. Therefore, it would be interesting to see if this can be exploited in order to speed-up computations. For instance, the decomposition of linear programs appears to be attractive in this context since the decomposition of matrix $A$ has to be done only once and could then be used in all iterations.

\section{References}

[1] Y. Censor. Binary steering in discrete tomography reconstruction with sequential and simultaneous iterative algorithms. Lin. Algebra and its Appl., 339:111-124, 2004.

[2] T. Pham Dinh and L.T. Hoai An. A d.c. optimization algorithm for solving the trust-region subproblem. SIAM J. Optim., 8(2): 476-505, 1998

[3] T. Pham Dinh and S. Elbernoussi. Duality in d.c. (difference of convex functions) optimization subgradient methods. In Trends in Mathematical Optimization, Int. Series of Numer. Math., volume 84, pages 277-292. Birkhäuser Verlag, Basel.

[4] P. Fishburn, P. Schwander, L. Shepp, and R. Vanderbei. The discrete radon transform and its approximate inversion via linear programming. Discr. Appl. Math., 75:39-61, 1997.

[5] R.J. Gardner and P. Gritzmann. Discrete tomography: Determination of finite sets by $x$ rays. Trans. Amer. Math. Soc., 349(6): 2271-2295, 1997

[6] P. Gritzmann, S. de Vries, and M. Wiegelmann. Approximating binary images from discrete $X$-rays. SIAM J. Optimization, 11(2):522-546, 2000.

[7] P. Gritzmann, D. Prangenberg, S. de Vries, and M. Wiegelmann Success and failure of certain reconstruction and uniqueness algorithms in discrete tomography. Int. J. Imag. Syst. Technol., 9:101-109, 1998. 
[8] G. Herman and A. Kuba. Discrete Tomography: Foundations, Algorithms, and Applications. Birkhäuser Boston, 1999.

[9] J.M. Kleinberg and E. Tardos. Approximation algorithms for classification problems with pairwise relationships: Metric labeling and Markov random fields. IEEE Symp. Foundations of Comp. Science, pages 14-23, 1999.

[10] A. Kuba and G.T. Herman. Discrete tomography: A historical overview. In G. T. Herman and A. Kuba, editors, Discrete Tomography: Foundations, Algorithms, and Applications, pages 3-34. Birkhäuser Boston, 1999.

[11] F. Natterer and F. Wübbeling. Mathematical Methods in Image Reconstruction. SIAM, Philadelphia, 2001

[12] R.T. Rockafellar. Convex analysis. Princeton Univ. Press, Princeton, NJ, 2 edition, 1972.

[13] S. Weber, C. Schnörr, and J. Hornegger. A linear programming relaxation for binary tomography with smoothness priors. In Proc. Int. Workshop on Combinatorial Image Analysis (IWCIA'03), 2003. Palermo, Italy, May 14-16/2003.

[14] S. Weber, T. Schüle, C. Schnörr, and J. Hornegger. A Linear Programming Approach to Limited Angle 3D Reconstruction from DSA Projections Special Issue of Methods of Information in Medicine, 4, 2004. (im press). 


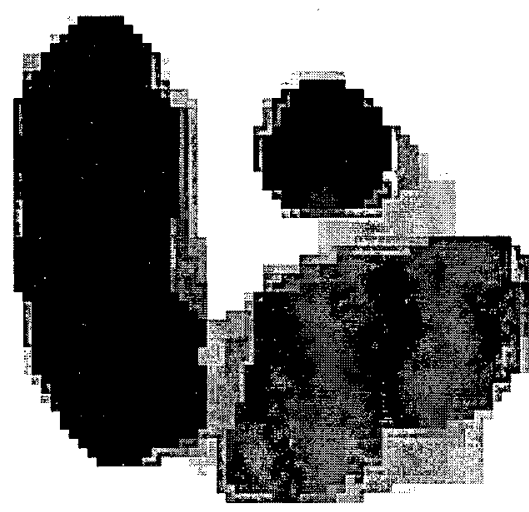

(a) Iteration 1 .

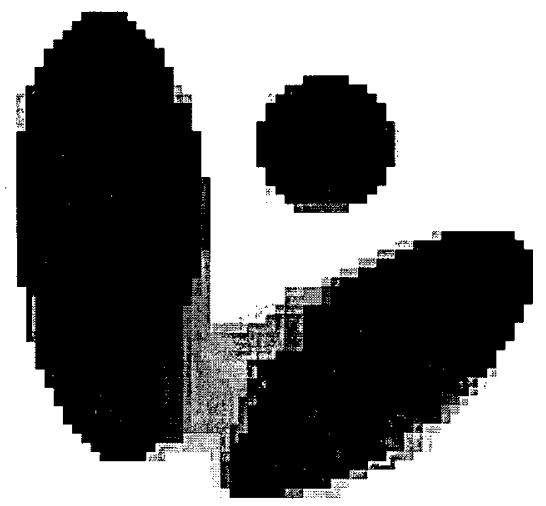

(c) Iteration 8 .

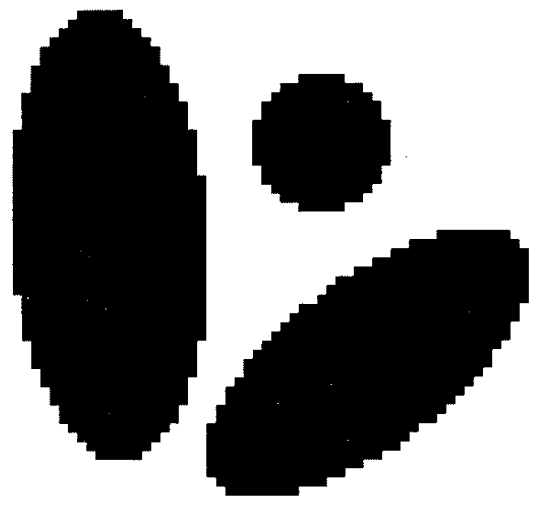

(e) Iteration 10 .

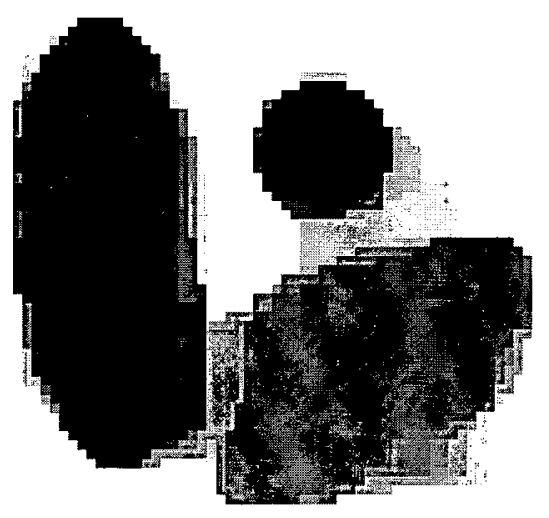

(b) Iteration 5 .

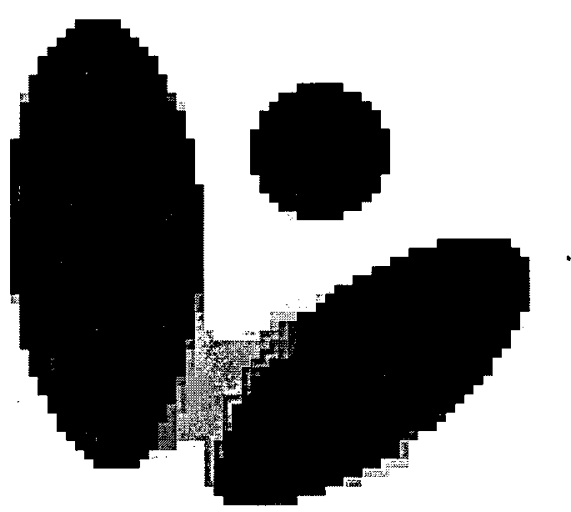

(d) Iteration 9.

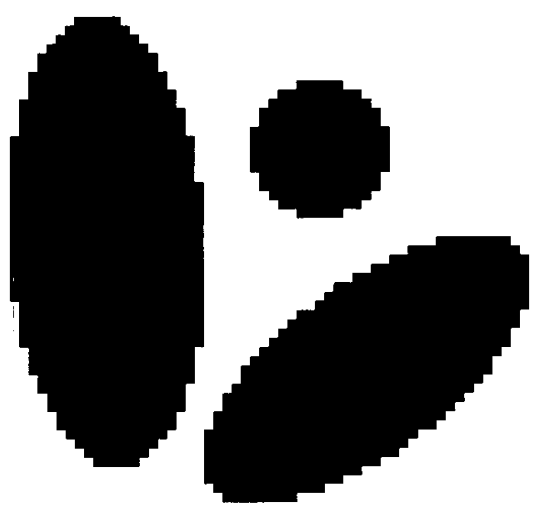

(f) Original

Figure 8. (a)-(e) Results at different iterations of our proposed reconstruction method. The original image is shown in figure $8(\mathrm{f})$ from which three projections, $0^{\circ}, 45^{\circ}$, and $90^{\circ}$ were taken. 


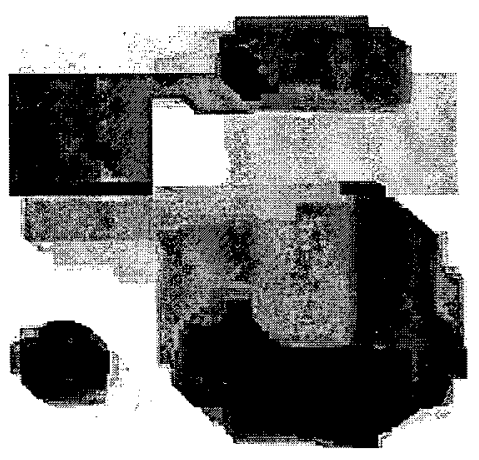

(a) Iteration 1 .

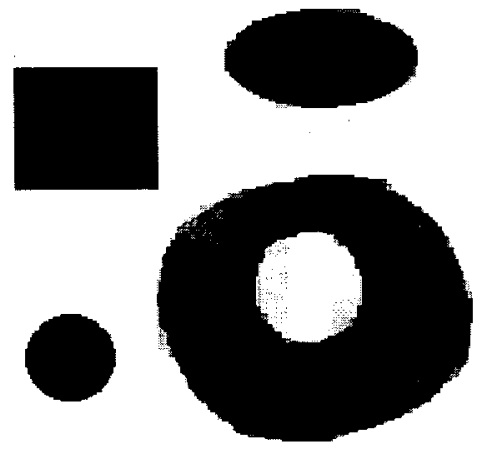

(c) Iteration 11.

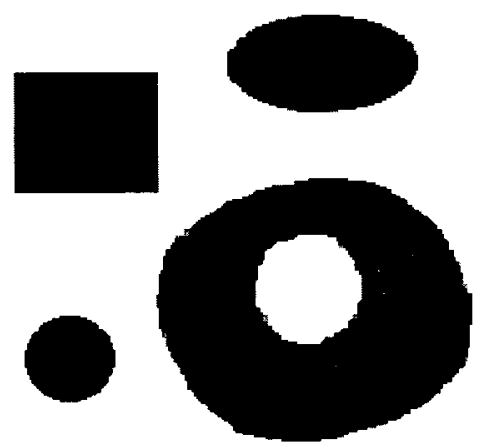

(e) Iteration 51.

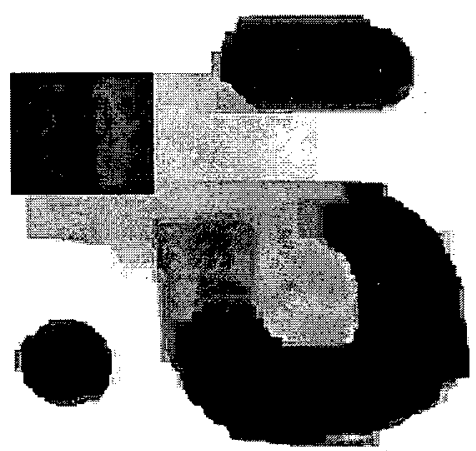

(b) Iteration 6.

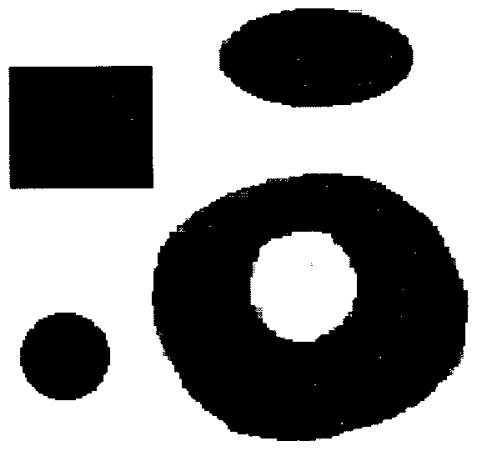

(d) Iteration 21 .

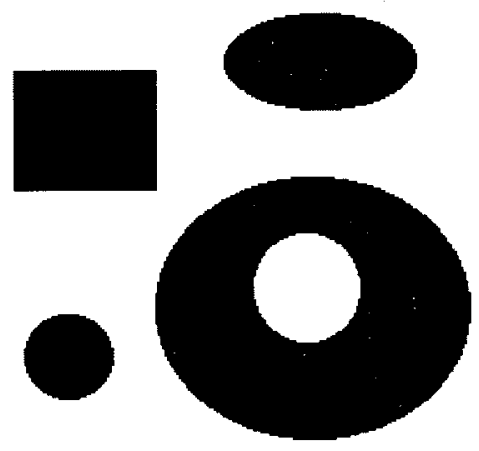

(f) Original.

Figure 9. (a)-(e) show the results at different iteration steps. The original image is shown in (f) from which three projections, $0^{\circ}, 45^{\circ}$, and $90^{\circ}$, were taken. 